http://dx.doi.org/10.11646/phytotaxa.112.1.4

\title{
Notes on Early Land Plants Today. 43. New combinations in Lophocoleaceae (Marchantiophyta)
}

\author{
LARS SÖDERSTRÖM ${ }^{1}$, JIŘÍ VÁŇA², BARBARA CRANDALL-STOTLER ${ }^{3}$, RAYMOND E. STOTLER ${ }^{3}$, \\ ANDERS HAGBORG ${ }^{4} \&$ MATT VON KONRAT $^{4}$ \\ ${ }^{I}$ Department of Biology, Norwegian University of Science and Technology, N-7491 Trondheim, Norway; lars.soderstrom@bio.ntnu.no \\ ${ }^{2}$ Department of Botany, Charles University, Benátská 2, CZ-12801 Praha 2, Czech Republic; vana@natur.cuni.cz \\ ${ }^{3}$ Department of Plant Biology, Southern Illinois University, Carbondale, Illinois 62901-6509, USA; crandall@ plant.siu.edu, \\ stotler@plant.siu.edu \\ ${ }^{4}$ Department of Botany, The Field Museum, 1400 South Lake Shore Drive, Chicago, IL 60605-2496, USA; hagborg@ pobox.com, \\ mvonkonrat@fieldmuseum.org
}

Söderström et al. (2013) outlined the current status of the family Lophocoleaceae. Following their concept several new combinations are needed, most of which are made here. While many of the taxa treated below need to be lectotypified, we are generally not doing that here.

We only transfer taxa that have been shown to belong to the new genus in recent studies (molecular and/or morphological) or taxa that have been studied by the authors (see Söderström et al. 2013 for further references). There still exist taxa that need to be transferred from Chiloscyphus, but we are not transferring them here, since it is unclear to us in which genus they belong.

The format of this note follows Söderström et al. (2012) except that we use the Melbourne International Code of Nomenclature for algae, fungi, and plants (ICN; McNeill et al. 2012) instead of the Vienna International Code of Botanical Nomenclature (ICBN; McNeill et al. 2006).

\section{New combinations in Cryptolophocolea}

Cryptolophocolea L.Söderstr., Crand.-Stotl., Stotler et Váňa, Phytotaxa 97: 39, 2013 (Söderström et al. 2013).

Type: Cryptolophocolea ciliolata (Nees) L.Söderstr., Crand.-Stotl., Stotler et Ván̆a, Phytotaxa 97: 39, 2013 (Söderström et al. 2013)

Note:- It is not fully clear which taxa to include in this genus and which to refer to Lophocolea or other genera. In this paper, we include only those taxa that molecular studies have confirmed to belong to Cryptolophocolea and taxa that recently have been classified into Chiloscyphus subgen. Connati (as well as other taxa that are morphologically close to it; cf. Söderström et al. 2013). The first group of taxa that follows are transferred based upon molecular evidence provided in Hentschel et al. (2007), Glenny et al. (2009), Engel et al. (2010), Engel \& He (2010), Vanderpoorten \& Long (2006) and Vanderpoorten et al. (2012) in addition to morphology.

Cryptolophocolea chiloscyphoidea (Lindenb. ex Lehm.) L.Söderstr. et Crand.-Stotl., comb. nov. Basionym:-Plagiochila chiloscyphoidea Lindenb. ex Lehm., Nov. Stirp. Pug. 8: 4, 1844. (Lehmann 1844).

Type:-CHILE. Magellanstr., Port Famine, Jacquinot 59a (lectotype [Grolle 1963: 65] PC).

$\equiv$ Chiloscyphus chiloscyphoideus (Lindenb. ex Lehm.) Vanderp., Schäf.-Verw. et D.G.Long, Taxon 59: 185, 2010

(Vanderpoorten et al. 2010). 三Leptoscyphus chiloscyphoideus (Lindenb. ex Lehm.) Gottsche, Bot. Zeitung (Berlin) 16, Beil.: 33, 1858 (Gottsche 1858).

Cryptolophocolea ciliolata (Nees) L.Söderstr., Crand.-Stotl., Stotler et Váňa, Phytotaxa 97: 39, 2013 (Söderström et al. 2013). 
Basionym:-Jungermannia ciliolata Nees, Enumer. Pl. Javae: 68, 1830 (Nees 1830).

Type:-INDONESIA. Java: in ligno putrido, rara, Blume, ex hb. Lehmann, misit Nees 1832, (syntype S-B25177).

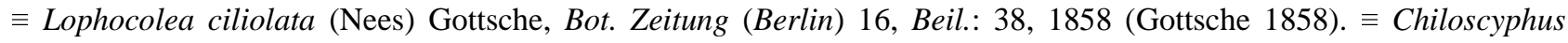
ciliolatus (Nees) J.J.Engel et R.M.Schust., Nova Hedwigia 39: 413, 1984 [1985] (Engel \& Schuster 1985).

= Lophocolea gaudichaudii Mont., Ann. Sci. Nat., sér. 2, 19: 250, 1843 (Montagne 1843), syn. fide Piippo (1985: 154). $\equiv$ Chiloscyphus gaudichaudii (Mont.) J.J.Engel et R.M.Schust., Nova Hedwigia 39: 415, 1984 [1985] (Engel \& Schuster 1985).

= Lophocolea beecheyana Taylor, London J. Bot. 5: 365, 1846 (Taylor 1846), syn. fide Piippo (1985: 154). $\equiv$ Chiloscyphus beecheyanus (Taylor) J.J.Engel et R.M.Schust., Nova Hedwigia 39: 411, 1984 [1985] (Engel \& Schuster 1985).

Cryptolophocolea compacta (Mitt.) L.Söderstr., comb. nov.

Basionym:-Lophocolea compacta Mitt., Trans. Linn. Soc. London, Bot. 3: 198, 1891 (Mitten 1891).

Type:-JAPAN, "Challenger Exp.", 1875 (NY).

= Lophocolea japonica Steph., Bull. Herb. Boissier sér. 2, 6: 941 (Sp. Hepat. 3: 121), 1906 (Stephani 1906c), syn. fide Inoue (1959: 222). $\equiv$ Chiloscyphus japonicus (Steph.) J.J.Engel et R.M.Schust., Nova Hedwigia 39: 417 , 1984 [1985] (Engel \& Schuster 1985), nom. illeg. (ICN Art. 53.1; non Chiloscyphus japonicus Steph., Bull. Herb. Boissier sér. 2, 7: 697 (Sp. Hepat. 3: 207), 1907 (Stephani 1907c). 三 Chiloscyphus yezoensis T.Katag. et Furuki, Bryol. Res. 10: 204, 2012 (Katagiri \& Furuki 2012). Note:-This name was recently proposed by Katagiri \& Furuki as a nom. nov. for the later homonym Chiloscyphus japonicus (Steph.) J.J.Engel et R.M.Schust. Chiloscyphus japonicus Steph. was reduced to Chiloscyphus polyanthos by Hattori (1952: 44).

= Lophocolea integristipula Steph., Bull. Herb. Boissier sér. 2, 6: 941 (Sp. Hepat. 3: 121), 1906 (Stephani $1906 \mathrm{c}$ ), syn. fide Hattori (1952:44). 三 Chiloscyphus integristipulus (Steph.) J.J.Engel et R.M.Schust., Nova Hedwigia 39: 417, 1984 [1985] (Engel \& Schuster 1985).

Cryptolophocolea connata (Sw.) L.Söderstr. et Váňa, comb. nov.

Basionym:-Jungermannia connata Sw., Prodr. (Swartz): 143, 1788 (Swartz 1788).

Type:-JAMAICA, Swartz (Lectotype [here designated] S-B25227!, isolectotypes G, NY-575464, S [5 specimens] UPS-B39480).

$\equiv$ Lophocolea connata (Sw.) Nees ex Mont., Ann. Sci. Nat., Bot. (sér. 2) 12: 51, 1839 (Montagne 1839). Chiloscyphus connatus (Sw.) J.J.Engel et R.M.Schust., Nova Hedwigia 39: 413, 1984 [1985] (Engel \& Schuster 1985).

Cryptolophocolea costata (Nees) L.Söderstr. comb. nov.

Basionym:- Jungermannia costata Nees, Enumer. Pl. Javae: 69, 1830 (Nees 1830).

Type:-INDONESIA. Habitat inter muscos locis montosis Javae, Blume, ex hb. Lehmann, (syntype S-B25269).

$\equiv$ Lophocolea costata (Nees) Gottsche, Bot. Zeitung (Berlin) 16, Beil.: 38, 1858 (Gottsche 1858). $\equiv$ Chiloscyphus costatus (Nees) J.J.Engel et R.M.Schust., Nova Hedwigia 39: 413, 1984 [1985] (Engel \& Schuster 1985).

=Lophocolea giulianettii Steph., Bull. Herb. Boissier sér. 2, 6: 953 (Sp. Hepat. 3: 133), 1906. (Stephani 1906c), syn. fide Piippo (1985: 156). 三 Chiloscyphus giulianettii (Steph.) J.J.Engel et R.M.Schust., Nova Hedwigia 39: 415,1984 [1985] (Engel \& Schuster 1985).

Cryptolophocolea guadalupensis (Steph.) L.Söderstr. et Váňa, comb. nov.

Basionym:-Lophocolea guadalupensis Steph., Bull. Herb. Boissier sér. 2, 7: 65 (Sp. Hepat. 3: 153 ), 1907 (Stephani 1907a).

Type:-GUADELOUPE. s.loc., Perrottet, Herb Stephani no. 1369 (lectotype [Fulford 1976: 448] G-00051406).

$\equiv$ Chiloscyphus guadalupensis (Steph.) J.J.Engel et R.M.Schust., Nova Hedwigia 39: 416, 1984 [1985] (Engel \& Schuster 1985), "guadeloupensis".

Cryptolophocolea helmsiana (Steph.) L.Söderstr., comb. nov.

Basionym:-Lophocolea helmsiana Steph., Bull. Herb. Boissier sér. 2, 6: 794 (Sp. Hepat. 3: 94), 1906 (Stephani $1906 a)$. Type:-NEW ZEALAND, s.loc., Helms, Herb. Stephani no. 17631 (G-00112467).

$\equiv$ Chiloscyphus helmsianus (Steph.) J.J.Engel et R.M.Schust., Nova Hedwigia 39: 416, 1984 [1985] (Engel \& Schuster 1985).

Cryptolophocolea leucophylla (Hook.f. et Taylor) L.Söderstr., comb. nov.

Basionym:-Jungermannia leucophylla Hook.f. et Taylor, London J. Bot. 3: 384, 1844 (Hooker \& Taylor 1844). 
Type:-NEW ZEALAND. Auckland Islands: Auckland Creek, 1840, Hooker (syntypes BM, E, FH-259486).

$\equiv$ Chiloscyphus leucophyllus (Hook.f. et Taylor) Taylor ex Gottsche, Lindenb. et Nees, Syn. Hepat. 2: 181 , 1845 (Gottsche et al. 1845).

= Chiloscyphus verrucosus (Steph.) J.J.Engel et R.M.Schust., Nova Hedwigia 39: 415, 1984 [1985] (Engel \& Schuster 1985), syn. fide Engel (1991a: 312).

= Chiloscyphus fulvus (Steph.) J.J.Engel et R.M.Schust., Nova Hedwigia 39: 425, 1984 [1985] (Engel \& Schuster 1985), syn. fide Engel (1991a: 312).

Cryptolophocolea martiana (Nees) L.Söderstr., Crand.-Stotl. \& Stotler, comb. nov., subsp. martiana Basionym:-Lophocolea martiana Nees, Syn. Hepat. 2: 152, 1845 (Gottsche et al. 1845).

Type:-BRAZIL. Martius (syntype W-Lindenb. Hep. 4092!).

$\equiv$ Chiloscyphus martianus (Nees) J.J.Engel et R.M.Schust., Nova Hedwigia 39: 419, 1984 [1985] (Engel \& Schuster 1985).

= Chiloscyphus jugalifolius Taylor, London. J. Bot. 5: 283, 1846. (Taylor 1846), syn. fide Fulford (1976: 441).

= Lophocolea congoana Steph., Bull. Herb. Boissier sér. 2, 7: 306 (Sp. Hepat. 3: 170), 1907. (Stephani 1907c), syn. fide Gradstein et al. (1983: 135 ). 三 Chiloscyphus congoanus (Steph.) J.J.Engel et R.M.Schust., Nova Hedwigia 39: 419, 1984 [1985] (Engel \& Schuster 1985).

= Lophocolea newtonii Steph., Bull. Herb. Boissier sér. 2, 7: 306 (Sp. Hepat. 3: 170), 1907 (Stephani 1907b), syn. nov. Type:-Africa occ. Insula Principe, Newton 11, Herb. Stephani no 17741 (lectotype [Jones 1953: 190] G-00045369). $\equiv$ Lophocolea martiana subsp. newtonii (Steph.) R.M.Schust., Hepat. Anthocerotae N. Amer. 4: 237, 1980 (Schuster 1980).

Cryptolophocolea martiana subsp. bidentula (Nees) L.Söderstr., Crand.-Stotl. \& Stotler, comb. nov. Basionym:-Chiloscyphus bidentulus Nees, Syn. Hepat. 2: 181, 1845 (Gottsche et al. 1845).

Type:-BRAZIL. Hb. Hampe.

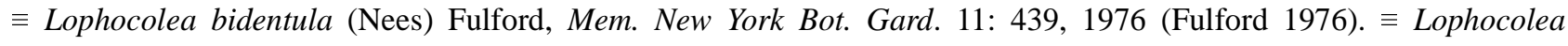
martiana subsp. bidentula (Nees) Gradst., Mem. New York Bot. Gard. 87: 73, 2003 (Gradstein \& Costa 2003).

Cryptolophocolea massalongoana (Schiffn.) L.Söderstr., comb nov.

Basionym:-Lophocolea massalongoana Schiffn., Akad. Wiss. Wien, Math.-Naturwiss. Kl., Denkschr. 70: 198, 1900 (Schiffner 1900).

Type:-Needs to be lectotypified from the many syntypes in FH.

三 Chiloscyphus costatoides J.J.Engel et R.M.Schust., Nova Hedwigia 39: 413, 1984 [1985] (Engel \& Schuster 1985), non Chiloscyphus massalongoanus Steph., Hedwigia 32: 325, 1893 (Stephani 1893).

Cryptolophocolea pallidovirens (Hook.f. et Taylor) L.Söderstr., comb. nov.

Basionym:- Jungermannia pallidovirens Hook.f. et Taylor, London J. Bot. 3: 473, 1844 (Hooker \& Taylor 1844).

Type:-CHILE. Tierra del Fuego: Cape Horn, Hooker 12 (lectotype [Vanderpoorten et al. 2010: 185] BM, isolectotypes G, NY).

$\equiv$ Chiloscyphus pallido-virens (Hook.f. et Taylor) Taylor ex Gottsche, Lindenb. et Nees, Syn. Hepat. 2: 178, 1845 (Gottsche et al. 1845), "pallide-virens".

Cryptolophocolea perissodonta (Spruce) L.Söderstr., comb. nov.

Basionym:-Lophocolea martiana var. perissodonta Spruce, Trans. \& Proc. Bot. Soc. Edinburgh 15: 432 , 1885 (Spruce 1885).

Type:-BRAZIL "fl. Negro et Uaupés; etiam circa Pará", Spruce (MANCH).

$\equiv$ Chiloscyphus perissodontus (Spruce) J.J.Engel et R.M.Schust., Nova Hedwigia 39: 421, 1984 [1985] (Engel \& Schuster 1985).

Note:-This transfer follows the study of Hentschel et al. (2007) but as indicated in that work (cf. their fig. 1) it may perhaps be better treated as an infraspecific taxon of Cryptolophocolea martiana.

Cryptolophocolea spinifera (Hook.f. et Taylor) L.Söderstr., comb. nov.

Basionym:-Jungermannia spinifera Hook.f. et Taylor, London J. Bot. 3: 381, 1844 (Hooker \& Taylor 1844).

Type:-NEW ZEALAND. Auckland Island: November 1840, Hooker (lectotype [Engel 2010: 128] FH, isolectotypes $\mathrm{BM})$.

$\equiv$ Chiloscyphus spinifer (Spruce) J.J.Engel et R.M.Schust., Nova Hedwigia 39: 423, 1984 [1985] (Engel \& Schuster 1985). 
The following taxa have been placed into Chiloscyphus subgen. Connati or are related to a species placed there, but have not yet been included in any molecular study. Morphology supports their transfer to Cryptolophocolea.

Cryptolophocolea aculeata (Mitt.) L.Söderstr., comb. nov.

Basionym:-Chiloscyphus aculeatus Mitt. in Hooker, Bot. Antarct. Voy. II (Fl. Nov.-Zel. 2): 140, 1854 (Mitten 1854).

Type:-NEW ZEALAND. "(herb. Mitten)" (NY, WELT).

Cryptolophocolea connatifolia (J.J.Engel) L.Söderstr., comb. nov.

Basionym:-Chiloscyphus connatifolius J.J.Engel, Phytologia 83: 42, 1997 [1998] (Engel 1998).

Type:-AUSTRALIA. Tasmania: Gordon River, Gorge Creek, near Pine Landing, sea level, Engel 14648 (holotype FC0000109F, isotype HO).

Cryptolophocolea edentata (J.J.Engel) L.Söderstr., comb. nov.

Basionym:-Chiloscyphus edentatus J.J.Engel, Phytologia 83: 43, 1997 [1998] (Engel 1998).

Type:-AUSTRALIA. Tasmania: Cradle Mtn.-Lake St. Clair Natl. Park, Ballroom Forest, SW side of Lake Dove, 9501050 m, Engel 13993 (holotype F-C0000108F, isotype HO).

Cryptolophocolea fleischeri (Steph.) L.Söderstr., comb. nov.

Basionym:-Lophocolea fleischeri Steph., Bull. Herb. Boissier sér. 2, 6: 952 (Sp. Hepat. 3: 132), 1906 (Stephani $1906 \mathrm{c})$.

Type:-SRI LANKA. Hunasgirias Peak, 9 February 1898, M. Fleischer ex herb. Levier 2029 (lectotype [here designated $]$ G-112452 [=G-17703]).

Note:-N. Kitagawa annotated all four syntypes in G in 1969. Three of them are annotated as synonyms of Lophocolea ciliolata (Nees) Steph. but the specimen cited above was annotated as "type". It is a packet containing only two stems.

$\equiv$ Chiloscyphus ceylonensis J.J.Engel, Fieldiana, Bot. n.s. 48: 133, 2010 (Engel 2010), non Chiloscyphus fleischeri Steph., Sp. Hepat. (Stephani) 6: 306, 1922 (Stephani 1922).

Cryptolophocolea levieri (Schiffn.) L.Söderstr., comb. nov.

Basionym:-Lophocolea levierii Schiffn., Akad. Wiss. Wien, Math.-Naturwiss. Kl., Denkschr. 70: 198, 1900 (Schiffner 1900)

Type:-INDONESIA. Java, Prov. Preanger: in silvis primaevis secus viam ad locum dictum "Tjiburrum" prope Tjibodas. 28.IV.1894 (c. per. et male), alt. 1575 m, Schiffner 1207 (lectotype [Piippo 1985: 160] FH-258724).

$\equiv$ Chiloscyphus paroicus J.J.Engel et R.M.Schust., Nova Hedwigia 39: 420, 1984 [1985] (Engel \& Schuster 1985), non Chiloscyphus levieri Steph., Bull. Herb. Boissier sér. 2, 8: 132 (Sp. Hepat. 3: 248), 1908 (Stephani 1908).

Cryptolophocolea lilliena (Steph.) L.Söderstr., comb. nov.

Basionym:-Lophocolea lilliena Steph., Sp. Hepat. (Stephani) 6: 282, 1922 (Stephani 1922).

Type:-KENYA. Near Mt. Kenia, 1910, A. G. Allan, Herb. Stephani no. 587 (G-00045366).

三 Chiloscyphus lillienus (Steph.) J.J.Engel et R.M.Schust., Nova Hedwigia 39: 418, 1984 [1985] (Engel \& Schuster 1985).

Cryptolophocolea mitteniana (Colenso) L.Söderstr., comb. nov., var. mitteniana

Basionym: Isotachis mitteniana Colenso, Trans. \& Proc. New Zealand Inst. 21: 69, 1888 [1889] (Colenso 1889).

Type:-NEW ZEALAND. North Is.: Waipawa Co., Ourisia slip, Redclyffe River, near Norsewood, 1885, Colenso a. 1300 (lectotype [Engel 1991a: 312] WELT, isolectotype BM).

三Chiloscyphus mittenianus (Colenso) J.J.Engel, Bryologist 94: 312, 1991. (Engel 1991a).

= Chiloscyphus vulcanicus Colenso, Trans. \& Proc. New Zealand Inst. 21: 59, 1888 [1889] (Colenso 1889), syn. fide Engel (1991a: 312).

= Chiloscyphus hispidus Steph., Sp. Hepat. (Stephani) 6: 308, 1922 (Stephani 1922), syn. fide Engel (2010: 175).

Cryptolophocolea mitteniana var. obtusa (J.J.Engel) L.Söderstr., comb. nov.

Basionym:-Chiloscyphus mittenianus var. obtusus J.J.Engel, Phytologia 83: 44, 1997 [1998] (Engel 1998).

Type:-NEW ZEALAND. South Is: Otago Prov.: Mt. Maungatua, W of Mosgiel, 760 m, Engel 17768 (holotype F, isotype CHR). 
Cryptolophocolea mitteniana var. symmetrica (J.J.Engel) L.Söderstr., comb. nov.

Basionym:-Chiloscyphus mittenianus var. symmetricus J.J.Engel, Phytologia 83: 44, 1997 [1998] (Engel 1998).

Type:-NEW ZEALAND. South Is., Westland Prov.: Westland Natl. Park, track to Alex Knob, off track to Louisa Peak, 1170 m, Engel 18973 (holotype F-C0000115F, isotype CHR).

Cryptolophocolea pallida (Mitt.) L.Söderstr., comb. nov.

Basionym:-Lophocolea pallida Mitt. in Hooker, Bot. Antarct. Voy. II (Fl. Nov.-Zel. 2): 135, 1854 (Mitten 1854).

Type:-NEW ZEALAND. "Northern Island, Auckland, Sinclair" (NY).

$\equiv$ Chiloscyphus pallidus (Mitt.) J.J.Engel et R.M.Schust., Nova Hedwigia 39: 420, 1984 [1985] (Engel \& Schuster 1985).

= Jungermannia multipenna Hook.f. et Taylor, London J. Bot. 4: 81, 1845, nom. illeg. (ICN Art. 53.1; hom. illeg. non

Hook.f. et Taylor 1844: 381) (Hooker \& Taylor 1845), syn. fide Mitten (1854: 135). $\equiv$ Chiloscyphus multipennus

J.J.Engel et R.M.Schust., Nova Hedwigia 39: 419, 1984 [1985] (Engel \& Schuster 1985).

Note:-When Hooker \& Taylor (1844) first described Jungermannia multipenna, they inadvertently repeated the diagnosis for Jungermannia intortifolia Hooker \& Taylor (1844: 374), which is the basionym of Isotachis intortifolia (Hook.f. et Taylor) Gottsche (1864: 121; see Hooker \& Taylor 1845: 81 where this error was first discussed). This makes Jungermannia multipenna 1844 (based on Campbell I. material) a superfluous name (nom. illeg. ICN Art. 52.1) since it was based on the same type as Jungermannia intortifolia. Aware of their error, the following year Hooker and Taylor published Jungermannia multipenna Hooker \& Taylor (1845: 81), based upon Auckland Is. material, but this is also illegitimate as a later homonym (ICN Art. 53.1) since Jungermannia multipenna 1844 had already been effectively published. When Gottsche et al. (1845) transferred Jungermannia multipenna to Lophocolea they cited the 1844 publication, technically making this name a synonym to Isotachis intortifolia. In Gottsche et al. (1847) they tried to correct it with a new combination citing the 1845 basionym, but as the earlier Lophocolea multipenna 1845 was effectively published, this later name also became an illegitimate homonym. In 1854 Mitten named Lophocolea pallida to include the nom. illeg. "[Jungermannia] Lophocolea multipenna Hook. fil et Tayl. Flor Antarct. p.155" as a synonym, noting that the Auckland Island specimen in Hooker's herbarium is a mix of vegetative shoots of Leioscyphus decipiens and a fertile shoot of Lophocolea pallida, and that the 1845 description of Jungermannia multipenna was compiled from both taxa. When Engel \& Schuster (1985) transferred Jungermannia multipenna 1845 to Chiloscyphus, it could have been considered a valid nom. nov. for all illegitimate names based on Jungermannia multipenna 1845 following ICN Art. 58.1 except for the fact that another epithet is available that has priority at that rank, namely Lophocolea pallida Mitt. When Engel (1991b) selected a lectotype for Jungermannia multipenna 1845 of Hooker plants from Auckland Is., he also reduced $L$. pallida to it, and in fact, all of the illustrations for Chiloscyphus multipennus used in his publication were from the type of Lophocolea pallida. Based on ICN Art. 58.1, this earlier epithet is the correct name for this taxon and is the basionym for the new combination above.

= Chiloscyphus involucratus Colenso, Trans. \& Proc. New Zealand Inst. 22: 455, 1890 (Colenso 1890) "involucrata", syn. fide Engel (2010: 137).

= Lophocolea erectifolia Steph., J. Linn. Soc., Bot. 29: 269, 1893 [1892] (Stephani 1892), syn. fide Hodgson (1953: 345). ?= Chiloscyphus erectifolius Steph., Bull. Herb. Boissier sér. 2, 8: 147 (Spec. Hep. 3: 263), 1908 (Stephani 1908), nom. inval. (ICN Art. 38.1(a); no description and ICN Art. 41.5; no basionym cited). Note:-The name "Chiloscyphus erectifolius St." was never validly published by Stephani but appears only in a list of taxa that he associated with the genus Lophocolea.

Cryptolophocolea proteus (Herzog) L.Söderstr., comb. nov.

Basionym:-Lophocolea proteus Herzog, Feddes Repert. Spec. Nov. Regni Veget. 57: 164, 1955 (Herzog 1955).

Type:-COLOMBIA. Killip 15330 (holotype JE-4001801).

$\equiv$ Chiloscyphus proteus (Herzog) J.J.Engel et R.M.Schust., Nova Hedwigia 39: 421, 1984 [1985] (Engel \& Schuster 1985).

Cryptolophocolea pycnophylla (Spruce) L.Söderstr., comb. nov.

Basionym:-Lophocolea pycnophylla Spruce, Trans. \& Proc. Bot. Soc. Edinburgh 15: 434, 1885 (Spruce 1885).

Type:-PERU. Mt. Guayrapurina, Spruce (syntype NY-1020409)

$\equiv$ Chiloscyphus pycnophyllus (Spruce) J.J.Engel et R.M.Schust., Nova Hedwigia 39: 421, 1984 [1985] (Engel \& Schuster 1985). 
Cryptolophocolea regularis (Steph.) L.Söderstr., comb. nov.

Basionym:-Chiloscyphus regularis Steph., Hedwigia 32: 325, 1893 (Stephani 1893).

Type:-RÉUNION. St. Pala, Rivière de Roches, Paul Lepervanche comm. clar. Bescherelle, Herb. Stephani no. 633 (G00045025).

= Lophocolea onraedtii Grolle, J. Hattori Bot. Lab. 55: 505, 1984 (Grolle 1984), syn. fide Engel (2010: 133).

Cryptolophocolea stephanii (Schiffn.) L.Söderstr., comb. nov.

Basionym:-Lophocolea stephanii Schiffn., Akad. Wiss. Wien, Math.-Naturwiss. Kl., Denkschr. 70: 197, 1900 (Schiffner 1900).

Type:-INDONESIA. Java: oberhalb Tjibodas, 1500-1800m; Pangerango oberhalb Tjiburrum, 1780m, Schiffner (FH; many syntypes, lectotypification needed).

三 Chiloscyphus ciliolatoides J.J.Engel et R.M.Schust., Nova Hedwigia 39: 412, 1984 [1985] (Engel \& Schuster 1985), non Chiloscyphus stephanii N.Kitag., J. Hattori Bot. Lab. 37: 266, 1973 (Kitagawa 1973).

Cryptolophocolea subopposita (J.J.Engel) L.Söderstr., comb. nov.

Basionym:-Chiloscyphus suboppositus J.J.Engel, Phytologia 83: 45, 1997 [1998] (Engel 1998).

Type:-AUSTRALIA. Tasmania: Cradle Mt.-Lake St. Clair Natl. Park, Pine Valley, Cephissus Falls, NNW of L. St. Clair, 850 m, Engel 14247 - c. sporo. (holotype F-C0000546F, isotype HO).

Cryptolophocolea thermarum (Schiffn.) L.Söderstr., comb. nov.

Basionym:-Lophocolea thermarum Schiffn., Akad. Wiss. Wien, Math.-Naturwiss. Kl., Denkschr. 70: 197, 1900 (Schiffner 1900).

Type:-INDONESIA. Java: oberhalb Tjiburrum am Pangerango, 2140 m, 2 May 1894, Schiffner, n. Q1197 (FH258729).

$\equiv$ Chiloscyphus thermarum (Schiffn.) J.J.Engel et R.M.Schust., Nova Hedwigia 39: 424, 1984 [1985] (Engel \& Schuster 1985).

Cryptolophocolea trialata (Gottsche) L.Söderstr., comb. nov.

Basionym:-Lophocolea trialata Gottsche, Linnaea 28: 552, 1856 [1857] (Gottsche 1857).

Type:-AUSTRALIA. Alpes australes, F. von Müller (L).

$\equiv$ Chiloscyphus trialatus (Gottsche) J.J.Engel et R.M.Schust., Nova Hedwigia 39: 410, 1984 [1985] (Engel \& Schuster 1985).

= Lophocolea tasmanica Mitt. in Hooker, Bot. Antarct. Voy. II (Fl. Tasman. 2): 226, 1859 (Mitten 1859), syn. fide Engel (2010: 147). $\equiv$ Chiloscyphus archeri J.J.Engel et R.M.Schust., Nova Hedwigia 39: 410, 1984 [1985] (Engel \& Schuster 1985) non Chiloscyphus tasmanicus Steph., Bull. Herb. Boissier sér. 2, 8: 133 (Sp. Hepat. 3: 249), 1908 (Stephani 1908).

$=$ Lophocolea bicuspidata Pearson, Bull. Misc. Inform. Kew 2: 71, 1924 (Pearson 1924), hom. illeg. (ICN Art. 53.1) non Lophocolea bicuspidata Steph., Sp. Hepat. (Stephani) 6: 263, 1922 (Stephani 1922), syn. fide Engel (2010: 147). $\equiv$ Chiloscyphus pearsonii J.J.Engel et R.M.Schust., Nova Hedwigia 39: 410, 1984 [1985] (Engel \& Schuster 1985), non Chiloscyphus bicuspidatus (Steph.) J.J.Engel et R.M.Schust., Nova Hedwigia 39: 411, 1984 [1985] (Engel \& Schuster 1985).

Cryptolophocolea tricorata (Hässel) Crand.-Stotl. et Stotler, comb. nov.

Basionym:-Chiloscyphus tricoratus Hässel, Nova Hedwigia 70: 456, 2000 (Hässel 2000a).

Type:-ARGENTINA. Tierra del Fuego: $1 \mathrm{~km} \mathrm{~S}$. of Paso Garibaldi. G. Hässel de Menéndez \& I. Gamundi 1480 (holotype BA).

Note:-This species is morphologically close to Cryptolophocolea pallidovirens but should be kept apart from that pending further study.

Cryptolophocolea tuberculata (J.J.Engel) L.Söderstr., comb. nov.

Basionym:-Chiloscyphus tuberculatus J.J.Engel, Phytologia 83: 45, 1997 [1998] (Engel 1998).

Type:-NEW ZEALAND. South Is., Southland Prov.: Fiordland Natl. Park, Tutoko River, W of Milford Sound, 50 m, Engel 18844 (holotype F-C0000107F, isotype CHR-558811). 
Cryptolophocolea whittieriana (Inoue et H.A.Mill.) L.Söderstr., comb. nov.

Basionym:-Lophocolea whittieriana Inoue et H.A.Mill., Bull. Natl. Sci. Mus., n.s. 8: 143, 1965 (Inoue \& Miller 1965).

Type:-MICRONESIA. Caroline Islands: Kusaie, Mt. Matante, 1800 ft, H.A. Miller \& H.O. Whittier 534 (syntypes BISH, G, MU, NY, TNS, US).

$\equiv$ Chiloscyphus whittierianus (Inoue et H.A.Mill.) J.J.Engel et R.M.Schust., Nova Hedwigia 39: 425, 1984 [1985] (Engel \& Schuster 1985).

\section{Transfer of taxa to Pachyglossa}

As outlined in Söderström et al. (2013), Lophocolea subgen. Notholophocolea should be included in Pachyglossa if not treated as a separate genus close to it.

Pachyglossa Herzog et Grolle, Rev. Bryol. Lichénol. 27: 150, 1958 [1959] (Herzog \& Grolle 1959). Type:-Pachyglossa tenacifolia (Hook.f. et Taylor) Herzog et Grolle.

= Lophocolea subgen. Notholophocolea R.M.Schust., Hepat. Anthocerotae N. Amer. 4: 183, 1980 (Schuster 1980), syn. nov.

Type:-Lophocolea boveana C.Massal.

$\equiv$ Chiloscyphus subg. Notholophocoleus (R.M.Schust.) J.J.Engel \& R.M.Schust., Nova Hedwigia 39: 410, 1984 [1985] (Engel \& Schuster 1985), "Notholophocolea".

= Chiloscyphus sect. Notholophocoleus J.J.Engel, J. Hattori Bot. Lab. 72 : 108 (Engel 1992), "Notholophocolea", nom. inval. (ICN Art. 38.1a; no description).

= Chiloscyphus sect. Notholophocoleus J.J.Engel, Fieldiana, Bot., n.s. 48: 12, 2010 (Engel 2010), syn. nov.

Type:-Chiloscyphus boveanus (C.Massal.) J.J.Engel et R.M.Schust.

Note:-When Engel (1992) described his sect. Gottscheoides, which he placed under subg. Notholophocoleus, he regarded sect. Notholophocoleus to be established as an autonym at the same time. However, the naming of sections within a subgenus does not explicitly create an autonym (ICN Art. 22.1).

= Chiloscyphus sect. Gottscheoides J.J.Engel, J. Hattori Bot. Lab. 72: 107 (Engel 1992), syn. nov.

Type:-Chiloscyphus gottscheoides (Besch. et C. Massal.) J.J.Engel et R.M.Schust.

Pachyglossa austrigena (Hook.f. et Taylor) L.Söderstr., comb. nov., subsp. austrigena

Basionym:-Jungermannia austrigena Hook.f. et Taylor, London J. Bot. 3: 466, 1844 (Hooker \& Taylor 1844).

Type:-CHILE. Prov. Magellanes: I. Hermite, Hooker s. n. (lectotype [Hässel 1995: 404] BM!, isolectotypes NY!, SB24899!, W).

$\equiv$ Lophocolea austrigena (Hook.f. et Taylor) Gottsche, Lindenb. et Nees, Syn. Hepat. 5: 702, 1847 (Gottsche et al. 1847).

三Chiloscyphus austrigenus (Hook.f. et Taylor) J.J.Engel et R.M.Schust., Nova Hedwigia 39: 411, 1984 [1985] (Engel \& Schuster 1985).

Pachyglossa austrigena subsp. okaritana (Steph.) L.Söderstr., comb. nov.

Basionym:-Lophocolea okaritana Steph., Bull. Herb. Boissier sér. 2, 6: 785 (Sp. Hepat. 3: 85), 1906 (Stephani 1906a). Type:-NEW ZEALAND. Okarito. Kirk 592, Herb. Stephani no. 16057 (lectotype [here designated] G-00064405).

$\equiv$ Chiloscyphus okaritanus (Steph.) J.J.Engel et R.M.Schust., Nova Hedwigia 39: 420, 1984 [1985] (Engel \& Schuster 1985). $\equiv$ Chiloscyphus austrigenus subsp. okaritanus (Steph.) J.J.Engel, J. Hattori Bot. Lab. 72: 113, 1992 (Engel 1992).

= Chiloscyphus cordifolius (Steph.) J.J.Engel et R.M.Schust., Nova Hedwigia 39: 413, 1984 [1985] (Engel \& Schuster 1985) nom. illeg. (ICN Art. 53.1) non Chiloscyphus cordifolius Rodway, Pap. \& Proc. Roy. Soc. Tasmania 1915: 106, 1916 (Rodway 1916), syn. fide Engel (2010: 16).

Pachyglossa boveana (C.Massal.) L.Söderstr., comb. nov.

Basionym:-Lophocolea boveana C.Massal., Nuovo Giorn. Bot. Ital. 17: 225, 1885 (Massalongo 1885).

Type:-ARGENTINA. Tierra del Fuego: Staten Island, Mt. Conegliana, 600m, Spegazzini (syntypes G, LPS, VER).

$\equiv$ Chiloscyphus boveanus (C.Massal.) J.J.Engel et R.M.Schust., Nova Hedwigia 39: 411, 1984 [1985] (Engel \& Schuster 1985). 
Pachyglossa gottscheoides (Besch. et C.Massal.) L.Söderstr., comb. nov.

Basionym:-Lophocolea gottscheoides Besch. et C.Massal., Bull. Mens. Soc. Linn. Paris 1: 631, 1886 (Bescherelle \& Massalongo 1886).

Type:-CHILE. Magellanes: I. Hermite, Hariot 143 (syntypes FH!, G, VER).

$\equiv$ Chiloscyphus gottscheoides (Besch. et C.Massal.) J.J.Engel et R.M.Schust., Nova Hedwigia 39: 415, 1984 [1985] (Engel \& Schuster 1985).

Pachyglossa otiphylla (Hook.f. et Taylor) Ván̆a, comb. nov.

Basionym:- Jungermannia otiphylla Hook.f. et Taylor, London J. Bot. 3: 466, 1844 (Hooker \& Taylor 1844).

Type:-CHILE. Magellanes: Tierra del Fuego, I. Hermite, Hooker (syntypes NY, S, W).

三 Chiloscyphus otiphyllus (Hook.f. et Taylor) J.J.Engel et R.M.Schust., Nova Hedwigia 39: 420, 1984 [1985] (Engel \& Schuster 1985).

\section{New combinations in Lophocolea}

The genus Lophocolea was traditionally considered distinct from Chiloscyphus, based on morphological characters, until Engel \& Schuster (1985) united them. Although their concept has been accepted by some bryologists for about a quarter of a century, for reasons outlined in Söderström et al. (2013) a return to a narrower concept of Chiloscyphus is now justified. Although most of the combinations into Chiloscyphus made by Engel \& Schuster (1985) were from Lophocolea, the narrower circumscription of Chiloscyphus requires that some new transfers from Chiloscyphus to Lophocolea be made, as detailed below.

Lophocolea (Dumort.) Dumort., Recueil Observ. Jungerm.: 17, 1835 (Dumortier 1835).

Basionym:- Jungermannia sect. Lophocolea Dumort., Syll. Jungerm. Europ.: 59, 1831 (Dumortier 1831).

= Chiloscyphus sect. Semiteres J.J.Engel, Novon 9: 22, 1999 (Engel 1999), syn. nov.

Type:-Chiloscyphus semiteres (Lehm.) Lehm. et Lindenb.

= Chiloscyphus sect. Novarum-Zeelandiarum J.J.Engel, Novon 9: 22, 1999 (Engel 1999) “Novae-zeelandii", syn. nov.

Type:-Chiloscyphus novae-zeelandiae (Lehm. et Lindenb.) J.J.Engel et R.M.Schust.

= Chiloscyphus sect. Hemispini J.J.Engel, Novon 9: 23, 1999 (Engel 1999), syn. nov.

Type:-Chiloscyphus parvispineus J.J.Engel

= Chiloscyphus sect. Aperticaulis J.J.Engel, Fieldiana, Bot. n.s. 48: 119, 2010 (Engel 2010), syn. nov.

Type:-Chiloscyphus aperticaulis J.J.Engel.

= Chiloscyphus sect. Spinoscyphus J.J.Engel, Fieldiana, Bot. n.s. 48: 199, 2010 (Engel 2010), syn. nov.

Type:-Chiloscyphus trichocoleoides Glenny, J.J.Engel et Xiao L.He

Lophocolea anisoloba (J.J.Engel et Glenny) L.Söderstr., comb. nov.

Basionym:-Chiloscyphus anisolobus J.J.Engel et Glenny, Bryologist 111: 118, 2008 (Engel \& Glenny 2008).

Type:-NEW ZEALAND. Canterbury Ecological Prov.: Waimakariri River, gorge of Taipoiti River, $1050 \mathrm{~m}, 6$ November 2005, Glenny 9736 (holotype F, isotype CHR-583361).

Lophocolea aperticaulis (J.J.Engel) L.Söderstr., comb. nov.

Basionym:-Chiloscyphus aperticaulis J.J.Engel, J. Hattori Bot. Lab. 95: 229, 2004 (Engel 2004).

Type:-NEW ZEALAND. North Is.: Gisborne Prov., Urewera Natl. Park, Waikareiti Track between track entrance and Lake Ruapani, N of eastern extremity of Lake Waikaremoana, 650-920 m, Engel 20519 - c. per. (holotype F, isotype AK).

Lophocolea aphelophylla (Hässel) Váňa, comb. nov.

Basionym:-Chiloscyphus aphelophyllus Hässel, J. Hattori Bot. Lab. 98: 123, 2005 (Hässel 2005)

Type:-CHILE. Sierra Pelada, camino de Unión a Hueicolla, bosque de Mirtáceas, 7 mai 1965, Hässel de Menéndez 2194 (holotype BA-13328).

Lophocolea apophylla (Hässel) Ván̆a, comb. nov.

Basionym:-Chiloscyphus apophyllus Hässel, J. Hattori Bot. Lab. 98: 126, 2005 (Hässel 2005).

Type:-CHILE. Osorno: Puyehue, on moist trunk in shady rainforest round Rio Chanleufú, 360 m, 25 January 1947, Sparre (holotype UPS). 
Lophocolea canaliculata (Hook.f. et Taylor ex Gottsche, Lindenb. et Nees) Steph. var. concava (J.J.Engel) L.Söderstr., comb. nov.

Basionym:-Chiloscyphus canaliculatus var. concavus J.J.Engel, Fieldiana, Bot. n.s. 48: 107, 2010 (Engel 2010).

Type:-NEW ZEALAND. Stewart I.; Fern Gully Track at terminus of Kaipipi Road, Mill Creek, ca. 2 km W of Oban, 20-30 m, Engel 24081 (holotype F, isotype CHR).

Lophocolea erosa (J.J.Engel) L.Söderstr., comb. nov.

Basionym:-Chiloscyphus erosus J.J.Engel, Phytologia 83: 43, 1997 [1998] (Engel 1998).

Type:-NEW ZEALAND. North I.: South Auckland Prov., Plateau E of Waiotapu Valley, ca. 1800 ft., Allison 3569 (holotype CHR-547427).

Lophocolea fertilis (J.J.Engel) L.Söderstr., comb. nov.

Basionym:-Chiloscyphus fertilis J.J.Engel, Phytologia 83: 43, 1997 [1998] (Engel 1998).

Type:-AUSTRALIA. New South Wales: Lane Cove, Forsyth 60 as L. bridelii - c. sporo + $\hat{\jmath}$ (holotype NSW).

Lophocolea gollanii (Steph.) Váňa, comb. nov.

Basionym:-Chiloscyphus gollanii Steph., Bull. Herb. Boissier sér. 2, 7: 837 (Sp. Hepat. 3: 209), 1907 (Stephani 1907d).

Type:-INDIA. Himachal Pradesh: Mussoorie, 14 September 1900, Gollan 3828 (G)

Lophocolea hattorii (J.J.Engel) L.Söderstr., comb. nov.

Basionym:-Chiloscyphus hattorii J.J.Engel, J. Hattori Bot. Lab. 74: 29, 1993 (Engel 1993).

Type:-NEW ZEALAND. South I.: Fiordland Natl. Park, Doubtful Sound, Elizabeth Is., 50-150 ft., Schuster 52852 (holotype F, isotype CHR-558812).

Lophocolea mediinfrons (J.J.Engel et Braggins) L.Söderstr., comb. nov.

Basionym:-Chiloscyphus mediinfrons J.J.Engel et Braggins, Fieldiana, Bot. n.s. 48: 107, 2010 (Engel 2010).

Type:-NEW ZEALAND. Stewart Is.: Paterson Inlet, Ulva Island, Flagstaff Point, NE end of island, sea level to ca. 60 m, Engel, von Konrat and Braggins 24598 A (holotype F, isotype AK).

Lophocolea koponenii (Piippo) Váňa, comb. nov.

Basionym:-Chiloscyphus koponenii Piippo, Ann. Bot. Fenn. 35: 55, 1998 (Piippo 1998).

Type:-PAPUA NEW GUINEA. West Sepik Province: Frieda River prospecting area of Frieda Copper Co. Mt. Hartley 8 km N of Frieda Base Camp, 5 August 1981, Koponen 35338 (holotype H).

Lophocolea novae-zeelandiae (Lehm. et Lindenb.) Nees var. meridionalis (Steph.) L.Söderstr., comb. nov. Basionym: Lophocolea meridionalis Steph., Bull. Herb. Boissier sér. 2, 6: 888 (Sp. Hepat. 3: 113), 1906 (Stephani 1906b).

Type::-NEW ZEALAND. South I.: Waimate (Canterbury), May 1901, Beckett, Herb. Stephani no. 17650 (G-60987).

Note:-This also creates the autonym Lophocolea novae-zeelandiae var. novae-zeelandiae.

Lophocolea parvispinea (J.J.Engel) L.Söderstr., comb. nov.

Basionym:-Chiloscyphus parvispineus J.J.Engel, Phytologia 83: 44, 1997 [1998] (Engel 1998).

Type:-NEW ZEALAND. South Is., Otago Prov.: S side of Mt. Cargill, just below summit, N of Dunedin, ca. 2200 m, Engel 17563 (holotype F, isotype CHR-571127).

Lophocolea piacenzai (Gola) Váňa, comb. nov.

Basionym:-Lophozia piacenzai Gola, Atti Reale Accad. Sci. Torino 49: 759, 1914 (Gola 1914).

Type:-Lophozia piacenzai n. sp. No. 2 Kashmir, Valle Sind, prima catena a partire da Srinagar: Mummer-Gund, 20002100 m, 20.5.1913, Prof. Borelli, det. G. Gola (lectotype [here designated] FT ex TOM!).

$=$ Lophocolea himalayensis A.Srivast. et S.C.Srivast., Indian Geocalycaceae: 182, pl. 56, 2002 (Srivastava \& Srivastava 2002), syn. nov.

Type:-INDIA. Western Himalayas, on way to Valley of Flowers, ca. 4000 m, Srivastava et al. 4166/80 (holotype LWU). 
Lophocolea semiteres (Lehm.) Mitt. var. retusa (J.J.Engel) L.Söderstr., comb. nov.

Basionym:-Chiloscyphus semiteres var. retusus J.J.Engel, Phytologia 83: 44, 1997 [1998] (Engel 1998).

Type:-AAUSTRALIA. New South Wales: Murrumbidgee River, Rules Point, 37 km NW of Adaminaby, Streimann 7482 (holotype CBG).

Note:-This also creates the autonym Lophocolea semiteres var. semiteres.

Lophocolea subporosa Mitt. var. inflexifolia (Steph.) L.Söderstr., comb. nov.

Basionym:-Lophocolea inflexifolia Steph., Sp. Hepat. (Stephani) 6: 278, 1922 (Stephani 1922).

Type:-NEW ZEALAND. Pain's Limestone Hill, Wairaropa, 28 November 1910, Gray 209, Herb Stephani no. 17637 (lectotype [here designated] G-00060805). Note:-Engel (2010: 49) indicates the current lectotype as type but did not state "designated here" or an equivalent (cf. ICN Art. 7.10).

Note:-This also creates the autonym Lophocolea subporosa Mitt. var. subporosa.

Lophocolea trichocoleoides (Glenny, J.J.Engel et Xiao L.He) L.Söderstr., comb. nov.

Basionym:-Chiloscyphus trichocoleoides Glenny, J.J.Engel et Xiao L.He, J. Bryol. 31: 100, 2009 (Glenny et al. 2009).

Type:-NEW ZEALAND. Western Nelson Ecological Province, Marino Mountains, valley between Mt Owen and Mt Bell, $41^{\circ} 32.7 ' \mathrm{~S}, 172^{\circ} 33.1^{\prime} \mathrm{E}$, NZMS $260 \mathrm{M} 28$ 726622, $1440 \mathrm{~m}, 19$ December 2006, Glenny 9888 (holotype CHR505239 , isotypes $\mathrm{F}, \mathrm{H})$.

Lophocolea wacei (S.W.Arnell ex J.J.Engel et Váňa) Váňa et L.Söderstr., comb. nov.

Basionym:-Chiloscyphus wacei S.W.Arnell ex J.J.Engel et Váňa, Mem. New York Bot. Gard. 105: 48, 2013 (Váňa \& Engel 2013).

Type:-TRISTAN DA CUNHA. Gough I.: Crest of ridge to N. of Main Glen, alt. 600 ft., 1 January 1956, Wace $559 b$ (holotype BM!, isotype UPS!).

Lophocolea werthii (J.J.Engel et R.M.Schust.) Váňa et L.Söderstr., comb. nov.

Basionym:-Chiloscyphus werthii J.J.Engel et R.M.Schust., Nova Hedwigia 39: 425, 1984 [1985] (Engel \& Schuster 1985).

Type:-KERGUELEN ISLAND. 12 Sept.1902, E. Werth (syntype FH-286153); am Station-See, 28 June1902, E. Werth (syntype FH-286154).

$\equiv$ Lophocolea variabilis Schiffn., Drygalski, Deutsche Südpolar-Exped. 1901-1903, 8 (Bot.): 73, 1906 [Oct.] (Schiffner 1906), nom. illeg. (ICN Art 53.1), non Lophocolea variabilis Steph., Bull. Herb. Boissier sér. 2, 6: 874 (Sp. Hepat. 3: 99), 1906 [Sept.] (Stephani 1906b).

\section{Transfer of Chiloscyphus breutelii to Leptoscyphus}

Hentschel et al. (2006) showed that Chiloscyphus breutelii is nested within Leptoscyphus. A position outside Chiloscyphus is also confirmed by Glenny et al. (2009), but they did not include any Leptoscyphus in their study. The oldest name for the taxon is Lophocolea trapezoïdes, but it is not available in Chiloscyphus.

Leptoscyphus Mitt., Hooker's J. Bot. Kew Gard. Misc. 3: 358, 1851 (Mitten 1851).

Type:-Leptoscyphus liebmannianus (Lindenb. et Gottsche) Mitt. [=Leptoscyphus porphyrius (Nees) Grolle)].

= Chiloscyphus subgen. Eurychiloscyphus Hässel, Lindbergia 26: 38, 2001 [2000] (Hässel 2000b), syn. nov.

Type:-Chiloscyphus horizontalis Hook.

Leptoscyphus trapezö̈des (Mont.) L.Söderstr., comb. nov.

Basionym:-Lophocolea trapezoïdes Mont., Ann. Sci. Nat. Bot. (sér. 2) 19: 251, 1843 (Montagne 1843).

Type:-ANTILLES. Perrottet (PC-fusion274592).

= Lophocolea breutelii Gottsche, Syn. Hepat. 2: 154, 1845 (Gottsche et al. 1845), syn. fide Fulford (1976: 435). $\equiv$ Chiloscyphus breutelii (Gottsche) J.J.Engel et R.M.Schust., Nova Hedwigia 39: 412, 1984 [1985] (Engel \& Schuster 1985). 


\section{Transfer of Chiloscyphus gremmenii to Stolonivector}

Chiloscyphus gremmenii was described using a very broad concept of the genus Chiloscyphus (sensu Engel \& Schuster 1985). Molecular analyses of a relatively large number of taxa (e.g., Hentschel et al. 2006, 2007) show that within the broadly defined genus were included a number of separate clades, some of which corresponded to described genera (see Söderström et al. 2013). Unfortunately, no specimens of the small, morphologically specialized genus Stolonivector Engel (1991c: 80) have been included in any molecular study to date. However, we conclude that, with acceptance of a narrower circumscription of Chiloscyphus and the additional, better developed material of Chiloscyphus gremmenii now available, Chiloscyphus gremmenii should be transferred to Stolonivector as it agrees with this genus in all characteristics presented by Engel (1991c) except that stolons are not common and gynoecia are on leading shoots. This species is similar to Stolonivector fiordlandiae (Hodgson 1953: 340) Engel (1991c: 82) and Stolonivector obtusilobus Engel (2009: 337). The differences can be summarized as follows: stolons relatively rare, leaves heteromorphic, of very variable form, sometimes deeply bi-(tri-)lobed, basal part of the leaves bistratose, gemmae present, gynoecia on leading shoots, and perianth mouth entire. Moreover, Stolonivector gremmenii has larger cells (25-30 $\times 20-27 \mu \mathrm{m})$ than Stolonivector fiordlandiae. Thick-walled cells without trigones and acute leaf lobes separate Stolonivector gremmenii from Stolonivector obtusilobus.

Stolonivector gremmenii (Váňa) Váňa, comb. nov.

Basionym:-Chiloscyphus gremmenii Váňa, Cryptog. Bryol. 26: 81, 2005 (Váňa \& Gremmen 2005).

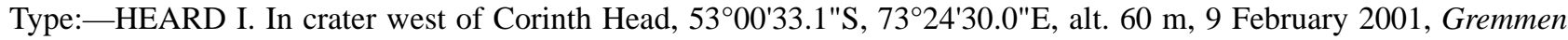
1524 (holotype ADT!, isotype PRC!).

\section{New synonym in Chiloscyphus Corda}

In Söderström et al. (2013) Chiloscyphus is circumscribed to include only taxa that can be assigned to Chiloscyphus subg. Chiloscyphus as defined morphologically by Schuster \& Engel (1985: 409). Relevant characters of the genus include the following: leaves unlobed; underleaves bifid, never connate with the leaves; perianth inflated to slightly prismatic, not sharply keeled; shoot calyptra well-developed, equal in length to the perianth or extending beyond it at maturity; androecia on leading stems, with the male bracts scarcely differentiated from the leaves. As currently defined, we think only four species belong to the genus.

Chiloscyphus kashyapii A.Srivast. et S.Srivast., Indian Geocalycaceae: 34, 2002 (Srivastava \& Srivastava 2002).

Type:-INDIA. West Bengal: Darjeeling, on way to Teesta Valley, alt. ca. 2000 m, 3 January 1970, Udar \& Srivastava 1757/70 (holotype LWU).

Chiloscyphus koeppensis (Gottsche) Steph., Bull. Herb. Boissier sér. 2, 8: 139 (Sp. Hepat. 3: 255), 1908 Stephani 1908).

Basionym:-Jungermannia koeppensis Gottsche in Neumayer, Int. Polarforsch., Deutsch. Exped. 2: 452, 1890 (Gottsche 1890).

Type:-SOUTH GEORGIA. Köppenberg, 10 February 1883, Will 11a (lectotype [Grolle 1972: 86] M, isolectotype HBG).

Chiloscyphus pallescens (Ehrh. ex Hoffm.) Dumort., Syll. Jungerm. Europ.: 67, 1831 (Dumortier 1831).

Basionym:-Jungermannia pallescens Ehrh. ex Hoffm., Deutschl. Fl., Theil 2 (Hoffm.): 87, 1795 [1796] (Hoffman 1796).

Type:-GERMANY. Harz, Ehrhart (lectotype [Grolle 1970: 646] MW [Hb. Hoffm. 8461] isolectotype [Ehrh. Plant. Crypt. Exs. no. 302. Hannover. 1793] LINN).

Note:-A var. fragilis (Roth) Müll.Frib. is occasionally recognized, sometimes even at the species level. 
=Chiloscyphus himalayensis Steph., Bull. Herb. Boissier sér. 2, 7: 837 (Spec. Hep. 3: 209), 1907 (Stephani 1907), syn. nov. Type:-INDIA. N. W. Himalaya, Dehra-Dun, prope Mussoorie, 6-7000', 2 January 1892, J. F. Duthie, Herb. Stephani no. 562 (lectotype [here designated] G-00069475, isolectotype JE-4005449!). Note:-The isolectotype of Chiloscyphus himalayensis in JE was annotated by R. Grolle as a synonym of Chiloscyphus pallescens but he never published it. The isolectotype consists of only two shoots originating from the richer specimen in $\mathrm{G}$.

Chiloscyphus polyanthos (L.) Corda, Naturalientausch 12: 651, 1829 (Corda 1829).

Basionym:-Jungermannia polyanthos L., Sp. Pl. ed. 1: 1131, 1753. (Linnaeus 1753).

Type:-GREAT BRITAIN. (lectotype [Grolle 1970: 646] OXF [specimen illustrated by Dillenius. 1741] isolectotype HSOL [Isoviita, Acta Bot. Fenn. 89: 17. 1970].

Note:-A var. rivularis (Schrad.) Lindb. et Arnell is occasionally recognized, sometimes even at the species level.

\section{Acknowledgement}

We thank M. Price (G) and L. Hedenäs (S) for help with type specimens. The Early Land Plants Today project (ELPT) has been generously supported in part by the Global Biological Information Facility (GBIF) Seed Money Award No.2007-41, activities facilitated in part by funding from the Biodiversity Synthesis Center of the Encyclopedia of Life (BioSynC), partial funding from the National Science Foundation (Award No's 0749762, 1115002, EF-0531750), the Warwick Foundation, and the Negaunee Foundation.

\section{References}

Bescherelle. E. \& Massalongo, C. (1886) Hepaticae novae americanae-australes. Bulletin mensuel de la société linnéenne de Paris 1: 626-640.

Colenso, W. (1889) A description of some newly-discovered cryptogamic plants; being a further contribution toward making known the botany of New Zealand. Transactions and proceedings of the New Zealand Institute 21 "1888": 43-80.

Colenso, W. (1890) A description of some newly-discovered and rare indigenous plants. Transactions and proceedings of the New Zealand Institute 22 "1889": 452-458.

Corda, A.J.C. (1829) Genera Hepaticarum. Die Gattungen der Lebermoose. In: Opiz, P.M. (Ed), Beiträge zur Naturgeschichte als Fortsetzung des Naturalientausches No. 12. Praha, pp. 643-655.

Dumortier, B.C. (1831) Sylloge Jungermannidearum Europae indigenarum, earum genera et species systematice complectens. J.Casterman, Tournay, $100 \mathrm{pp}$. http://dx.doi.org/10.5962/bhl.title.22343

Dumortier, B.C. (1835) Recueil d'Observations sur les Jungermanniacées. I. Révision des genres. J.-A.Blanquart, Tournay, $27 \mathrm{pp}$.

Engel, J.J. (1991a) Studies on Geocalycaceae (Hepaticae).5. Comments on the Leucophyllae complex of Chiloscyphus. Bryologist 94: 311-314. http://dx.doi.org/10.2307/3243840

Engel, J.J. (1991b) Studies on Geocalycaceae (Hepaticae). 3. On the typification of Jungermannia multipenna Hook. f. and Tayl. Bryologist 94: 22-24.

Engel, J.J. (1991c) Studies on Geocalycaceae (Hepaticae). II. Stolonivector, a new genus from New Zealand. Journal of the Hattori Botanical Laboratory 69: 79-86.

Engel, J.J. (1992) Studies on Geocalycaceae (Hepaticae). VIII. A revision of Chiloscyphus subg. Notholophocolea (Schust.) Engel \& Schust. Journal of the Hattori Botanical Laboratory 72: 105-115.

Engel, J.J. (1993) Studies in Geocalycaceae. IX. Chiloscyphus hattorii Engel, a new species from New Zealand, together with nomenclature refinements in Australasian Heteroscyphus and Leptoscyphus. Journal of the Hattori Botanical Laboratory 74: 29-33.

Engel, J.J. (1998 “1997”) Studies on Geocalycaceae (Hepaticae). X. New taxa and new combinations in Chiloscyphus Corda for Australasia. Phytologia 83: 42-46.

Engel, J.J. (1999) Studies on Geocalycaceae (Hepaticae). XI. Supraspecific new taxa and new combinations in Chiloscyphus Corda for Australia. Novon 9: 22-24.

Engel, J.J. (2004) Studies on Geocalycaceae. XV. Chiloscyphus aperticaulis Engel, an interesting new species of hepaticae from New Zealand. Journal of the Hattori Botanical Laboratory 95: 229-234. 
Engel, J.J. (2009) Studies on Lophocoleaceae (Hepaticae). XVIII. Stolonivector Engel in New Zealand, including two new species, together with comments on generic endemism of Austral Hepaticae. Nova Hedwigia 88: 335-346. http://dx.doi.org/10.1127/0029-5035/2009/0088-0335

Engel, J.J. (2010) Austral Hepaticae 45. A monograph of the genus Chiloscyphus Corda (Lophocoleaceae) for Australasia. Fieldiana, Botany, n.s. 48: 1-209. http://dx.doi.org/10.3158/0015-0746-48.1.1

Engel, J.J. \& Glenny, D. (2008) Studies on Lophocoleaceae XVI. Chiloscyphus anisolobus, an interesting new species from New Zealand. Bryologist 111: 118-123. http://dx.doi.org/10.1639/0007-2745(2008)111[118:solxca]2.0.co;2

Engel, J.J. \& He, X.-L. (2010) Studies on Lophocoleaceae. XIX. The systematic identity of Cyanolophocolea R.M.Schust., an intriguing liverwort from New Zealand and Australia, based on morphological and molecular evidence. Bryologist 113: 149-163. http://dx.doi.org/10.1639/0007-2745-113.1.149

Engel, J.J. \& Schuster, R.M. (1985 “1984”) An overview and evaluation of the genera of Geocalycaceae subfamily Lophocoleoideae (Hepaticae). Nova Hedwigia 39: 385-463.

Engel, J.J., He, X.-L. \& Glenny, D. (2010) Studies on Lophocoleaceae XXII. The systematic position of Amphilophocolea R.M.Schust. together with comments on the status of Tetracymbaliella Grolle and Lamellocolea R.M.Schust. Phytotaxa 9: 41-52.

Fulford, M. (1976) Manual of the leafy Hepaticae of Latin America IV. Memoirs of the New York Botanical Garden 11: $395-535$.

Glenny, D., Engel, J.J. \& He-Nygrén, X. (2009) The systematic identity of Chiloscyphus trichocoleoides, a new liverwort species from New Zealand, uncovered by morphological and molecular evidence. Journal of Bryology 31: 93-105. http://dx.doi.org/10.1179/174328209x427524

Gola, G. (1914) Epatiche del Kashmir raccolte dalla Spedizione Piacenza. Atti della Reale Accademia della Scienze di Torino. Classe di Scienze, Fisiche, Matematiche e Naturali 49: 757-761. http://dx.doi.org/10.1007/bf02960932

Gottsche, C.M. (1857 “1856”) Plantae Muellerianae: Hepaticae Australiae a Dre. Ferd. Müller lectae. Linnaea 28: 547561.

Gottsche, C.M. (1858) Übersicht und kritische Würdigung der seit dem Erscheinen der Synopsis Hepaticarum bekannt gewordenen Leistungen in der Hepaticologie. Botanische Zeitung (Berlin)16, Beilage: 1-48.

Gottsche, C.M. (1864) Hepaticae (in J Triana \& JE Planchon, Prodromus Florae Novo-Granatensis). Annales des Sciences Naturelles, Botanique, sér. 5. 1: 95-198.

Gottsche, C.M. (1890) Die Lebermoose Süd-Georgiens. In: Neumayer, G.B. (Ed), Die Internationale Polarforschung 1882-83. Die Deutsche Expeditionen und ihre Ergebnisse. II Beschreibende Naturwissenschaften. A. Asher \& Co.: Berlin, pp. 449-454.

Gottsche, C.M., Lindenberg, J.B.G. \& Nees von Esenbeck, C.G. (1845) Synopsis Hepaticarum, fasc. 2. Meissner, Hamburg, pp. 145-304. http://dx.doi.org/10.5962/bhl.title.15221

Gottsche, C.M., Lindenberg, J.B.G. \& Nees von Esenbeck, C.G. (1847) Synopsis Hepaticarum, fasc. 5. Meissner, Hamburg, pp. 625-834. http://dx.doi.org/10.5962/bhl.title.15221

Gradstein, S.R. \& Costa, D.P. (2003) The Hepaticae and Anthocerotae of Brazil. Memoirs of the New York Botanical Garden 87: 1-316.

Gradstein, S.R., Pócs, T. \& Ván̆a, J. (1983) Disjunct hepaticae in tropical America and Africa. Acta Botanica Academiae Scientiarum Hungarica 29: 127-171.

Grolle, R. (1963 “1962”) Monographie der Lebermoosgattung Leptoscyphus Mitt. Nova Acta Leopoldina. Abhandlungen der Kaiserlich Leopoldinisch-Carolinisch Deutschen Akademie der Naturforscher 25: 1-143.

Grolle, R. (1970) Nomen conservandum propositum (312). Chiloscyphus Corda. Taxon 19: 646-647.

Grolle, R. (1972) The hepatics of South Sandwich Islands and South Georgia. Bulletin, British Antarctic Survey 28: 8395.

Grolle, R. (1984) Miscellanea Hepaticologica 221-230. Journal of the Hattori Botanical Laboratory 55: 501-511.

Hässel de Menéndez, G.G. (1995) Little known Chiloscyphus species from southern South America (Hepatophyta). Nova Hedwigia 61: 39-415.

Hässel de Menéndez, G.G. (2000a) Parachiloscyphus Hässel, a new subgenus of Chiloscyphus Corda (Hepatophyta) from southern South America, including a new species. Nova Hedwigia 70: 451-460.

Hässel de Menéndez, G.G. (2000b “2001”) Eurychiloscyphus Hässel, a new subgenus of Chiloscyphus Corda (Hepatophyta) from southern South America. Lindbergia 26: 37-42.

Hässel de Menéndez, G.G. (2005) On Chiloscyphus aphelophyllus Hässel sp. nov. and C. apophyllus Hässel sp. nov. 
(Marchantiophyta, Geocalycaceae) from Chile. Journal of the Hattori Botanical Laboratory 98: 123-129.

Hattori, S. (1952) Hepaticae of Shikoku and Kyushu, southern Japan (1). Journal of the Hattori Botanical Laboratory 7 : 38-61.

Hentschel, J., Feldberg, K., Zündorf, H.-J., Hellwig, F.H., Schneider, H. \& Heinrichs, J. (2007) The systematic position of Pachyglossa and Clasmatocolea (Jungermanniopsida: Lophocoleaceae) inferred from nrDNA ITS sequences and morphology. Taxon 56: 1136-1142. http://dx.doi.org/10.2307/25065908

Hentschel, J., Wilson, R., Burghardt, M., Zündorf, H.-J., Schneider, H. \& Heinrichs, J. (2006) Reinstatement of Lophocoleaceae (Jungermanniopsida) based on chloroplast gene rbcL data: exploring the importance of female involucres for the systematics of Jungermanniales. Plant Systematics and Evolution 258: 211-226. http://dx.doi.org/10.1007/s00606-006-0408-y

Herzog, T. (1955) Hepaticae aus Columbia und Peru. Feddes Repertorium specierum novarum regni vegetabilis 57: 156203. http://dx.doi.org/10.1002/fedr.4880570105

Herzog T. \& Grolle, R. (1959 “1958”) Was ist Pachyglossa? Revue Bryologique et Lichénologique 27: 147-165.

Hodgson, E.A. (1953) New Zealand Hepaticae (Liverworts) - VII. A review of the N. Z. species of the genus Lophocolea with notes on Chiloscyphus. Transactions of the Royal Society of New Zealand 80: 329-358.

Hoffman, G.F. (1796) Deutschlands Flora oder Botanisches Taschenbuch für das Jahr 1795. Cryptogamie. Johan Jakob Palm, Erlangen, 200 pp.

Hooker, J.D. \& Taylor, T. (1844) Hepaticae Antarcticae; being characters and brief descriptions of the Hepaticae discovered in the southern circumpolar regions during the voyage of H.M. Discovery ships Erebus and Terror. I. Species of Lord Auckland's and Campbell's Islands. London Journal of Botany 3: 366-400.

Hooker, J.D. \& Taylor, T. (1845) Hepaticae Antarcticae, supplementum; or specific characters, with brief descriptions. of some additional species of the Hepaticae of the Antarctic regions, New Zealand, and Tasmania, together with a few from the Atlantic Islands and New Holland. London Journal of Botany 4: 79-97.

Inoue, H. (1959) A review of Japanese species of Lophocolea Dum. Journal of the Hattori Botanical Laboratory 21: 214-230.

Inoue, H. \& Miller, H.A (1965) Hepaticae from Kusaie, Caroline Islands. Bulletin of the National Science Museum, n.s. 8: 139-160.

Jones, E.W. (1953) African Hepatics V. Lophocolea, with notes on Chiloscyphus and Leptoscyphus. Transactions of the British Bryological Society 2: 172-202. http://dx.doi.org/10.1179/006813853804878038

Katagiri, T. \& Furuki, T. (2012) Checklist of Japanese liverworts and hornworts, 2012. Bryological Research 10: 193210.

Kitagawa, N. (1973) Miscellaneous notes on little-known species of Hepaticae, 26-50. Journal of the Hattori Botanical Laboratory 37: 263-273.

Massalongo, C. (1885) Epatiche raccolte alla Tierra del Fuoco dal Dott. C. Spegazzini nell'anno 1882. Nuovo Giornale Botanico Italiano 17: 201-277.

Massalongo, C. (1906) Epatiche della Repubblica Argentina raccolte dal Prof. C. Spegazzini. Atti dell'Accademia delle scienze mediche e naturali in Ferrara 80: 1-14.

McNeill, J., Barrie, F.R., Buck, W.R., Demoulin, V., Greuter, W., Hawksworth, D.L., Herendeen, P.S., Knapp, S., Marhold, K., Prado, J., Prud'homme van Reine, W.F., Smith, G.F., Wiersema, J.H. \& Turland, N.J. (2012) International Code of Nomenclature for algae, fungi and plants (Melbourne Code) adopted by the Eighteenth International Botanical Congress Melbourne, Australia, July 2011. Regnum Vegetabile 154: 1-240.

McNeill, J., Barrie, F.R., Burdet, H.M., Demoulin, V., Hawksworth, D.L., Marhold, K., Nicolson, D.H., Prado, J., Silva, P.C., Skog, J.E., Wiersema, J.H. \& Turland, N.J. (2006) International Code of Botanical Nomenclature (Vienna Code) adopted by the Seventeenth International Botanical Congress Vienna, Austria, July 2005. Regnum Vegetabile 146: $1-260$.

Mitten, W. (1851) Catalogue of cryptogamic plants collected by Professor W. Jameson in the vicinity of Quito. Hooker's journal of botany and the Kew garden miscellany 3: 351-361.

Mitten, W. (1854) Hepaticae. In: Hooker, J.D. (Ed), The Botany of the Antarctic Voyage of H. M. discovery ships Erebus and Terror in the years 1839-43. II. Flora Novae Zealandiae 2. Reeve: London, pp. 125-160. http://dx.doi.org/10.1111/j.1095-8339.1891.tb00626.x

Mitten, W. (1859) Hepaticae. In: Hooker, J.D. (Ed), The Botany of the Antarctic Voyage of H. M. discovery ships Erebus and Terror in the years 1839-43. III. Flora Tasmaniae 2. Reeve: London, pp. 221-241

Mitten, W. (1891) An Enumeration of all species of Musci and Hepaticae recorded from Japan. Transactions of the Linnean Society of London, Botany 3: 153-206.

Montagne, J.F.C. (1839) Cryptogamae Brasilienses seu Plantae cellulares quas in itinere per Brasiliam à celeb. Auguste de Sainte-Hilaire collectas recensuit observationibusque nonnullis illustravit. Annales des Sciences Naturelles, 
Botanique, sér. 2. 12: 42-55.

Montagne, J.F.C. (1843) Quatrième centurie de plantes cellulaires exotiques nouvelles, décades I-VI. Annales des Sciences Naturelles, Botanique, sér. 2. 19: 238-266.

Nees von Esenbeck, C.G. (1830) Enumeratio plantarum cryptogamicarum Javae et insularum adiacentium, quas a Blumio et Reinwardtio collectas describi edique curavit Christ. Godfr. Nees ab Esenbeck professor vratislaviensis. Fasciculus prior, hepaticas complectens, ab editore illustratis. Grass, Barth \& Co.: Breslau, 86 pp.

Pearson, W.H. (1924) Notes on Tasmanian hepatics. Bulletin of Miscellaneous Information, Royal Gardens, Kew 2: 66-75. http://dx.doi.org/10.2307/4118555

Piippo, S. (1985) Bryophyte flora of the Huon Peninsula, Papua New Guinea. XII. Geocalycaceae (Hepaticae). Acta Botanica Fennica 131: 129-167.

Piippo, S. (1998) Bryophytes from Frieda River, East and West Sepik Provinces, Papua New Guinea. IV. Chiloscyphus koponenii sp. nov. (Geocalycaceae). Annales Botanici Fennici 35: 55-57.

Rodway, L. (1916) Additions to the Tasmanian Flora. Papers and Proceedings of the Royal Society of Tasmania 1915: 104-107.

Schiffner, V. (1900) Exposito plantarum in itinere Suo Indico annis 1893/94 suspecto collectarum, II. Akademie der Wissenschaften in Wien. Mathematisch-Naturwissenschaftliche Klasse. Denkschriften 70: 155-218.

Schiffner, V. (1906) Die Lebermoose. In: Drygalski, E.D. (Ed), Deutsche Südpolar-Expedition 1901-1903, Band. 8 (Botanik). Georg Reimer: Berlin, pp. 59-80.

Schuster, R.M. (1980) The Hepaticae and Anthocerotae of North America. vol. IV. Columbia University Press: New York, 1334 pp.

Söderström, L., Hagborg, A. \& von Konrat, M. (2012) Notes on Early Land Plants Today. Phytotaxa 65: 41-42.

Söderström, L., Crandall-Stotler, B. Stotler, R., Váňa, J., Hagborg, A. \& von Konrat, M. (2013) Notes on Early Land Plants Today. 36. Generic treatment of Lophocoleaceae. Phytotaxa 97: 36-43. http://dx.doi.org/10.11646/phytotaxa.97.2.3

Spruce, R. (1885) Hepaticae Amazonica et Andinae. Transactions and Proceedings of the Botanical Society. Edinburgh 15: 309-588.

Srivastava, A. \& Srivastava, S.C. (2002) Indian Geocalycaceae (Hepaticae). A taxonomic study. Bishen Singh Madendra Pal Singh: Dehra Dun, 246 pp.

Stephani, F. (1892) A revision of Colenso's Hepaticae with descriptions of new species collected by him. Journal of the Linnean Society. Botany 29: 263-280. http://dx.doi.org/10.1111/j.1095-8339.1892.tb02036.x

Stephani, F. (1893) Hepaticarum species novae. Pars IV. Hedwigia 32: 315-327.

Stephani, F. (1906a) Species Hepaticarum 3. Bulletin de l'Herbier Boissier, série 2 6(9): 781-796.

Stephani, F. (1906b) Species Hepaticarum 3. Bulletin de l'Herbier Boissier, série 2 6(10): 874-889.

Stephani, F. (1906c) Species Hepaticarum 3. Bulletin de l'Herbier Boissier, série 2 6(11): 935-966.

Stephani, F. (1907a) Species Hepaticarum 3. Bulletin de l'Herbier Boissier, série 2 7(1): 59-72.

Stephani, F. (1907b) Species Hepaticarum 3. Bulletin de l'Herbier Boissier, série 2 7(4): 297-312.

Stephani, F. (1907c) Species Hepaticarum 3. Bulletin de l'Herbier Boissier, série 2 7(8): 683-698.

Stephani, F. (1907d) Species Hepaticarum 3. Bulletin de l'Herbier Boissier, série 2 7(10): 837-852.

Stephani, F. (1908) Species Hepaticarum 3. Bulletin de l'Herbier Boissier, série 2 8( 2): 125-148.

Stephani, F. (1922) Species Hepaticarum 6. George \& Cie: Genève \& Bale, pp. 241-368.

Swartz, O. (1788) Nova Genera et Species Plantarum, seu Prodromus descriptionum vegetabilium maximum partem incognitorum quae sub itinere in Indiam occidentalem annis 1783-1787. Uppsala, $152 \mathrm{pp}$. http://dx.doi.org/10.5962/bhl.title.4400

Taylor, T. (1846) New Hepaticae. London Journal of Botany 5: 258-284.

Ván̆a, J. \& Engel, J.J. (2013) The liverworts and hornworts of the Tristan da Cunha group. Memoirs of the New York Botanical Garden 105: 1-138.

Váňa, J. \& Gremmen, N. (2005) Hepatics of Heard Island. Cryptogamie, Bryologie 26: 79-90.

Vanderpoorten, A., Désamoré, A. Laenen, B \& Gradstein, S.R. 2012. Striking autapomorphic evolution in Physotheca J.J.Engel \& Gradst. (Marchantiophyta: Lophocoleaceae) blurred its actual relationships with Leptoscyphus Mitt. Journal of Bryology 34: 251-256. http://dx.doi.org/10.1179/1743282012y.0000000018

Vanderpoorten, A. \& Long, D.G. (2006). Budding speciation and neotropical origin of the Azorean endemic liverwort, Leptoscyphus azoricus. Molecular Phylogenetics and Evolution 40: 73-83. http://dx.doi.org/10.1016/j.ympev.2006.02.013

Vanderpoorten, A., Schäfer-Verwimp, A., Heinrichs, J., Devos, N. \& Long, D.G. (2010) The taxonomy of the leafy liverwort genus Leptoscyphus (Lophocoleaceae) revisited. Taxon 59: 176-186. 